\title{
Material Coding for Aircraft Manufacturing Industry
}

\author{
Hong Xia Cai ${ }^{1}$, Ming Yu Dai ${ }^{1}$, Tao Yu ${ }^{1}$
}

\begin{abstract}
Material coding is the basis for enterprises to perform information management. There are various kinds of materials in the aircraft manufacturing industry. In order to improve the efficiency of aircraft material management, this paper studies the aircraft material coding. The aircraft materials are divided into six categories based on some characteristics. The flexible material coding model is proposed consisting of code fields which indicate the material and code field relations in order to define the constraint relations among code fields. There are three kinds of code fields: class code, property code and flow code, while there are three kinds of relations, including the parallel relation, the subordinate relation and the dependency one. For convenient recognition and operation, the alpha-numeric combination code method is used. The material coding system in which the material code could be automatically generated was finally developed. The system has been applied in the aircraft manufacturing enterprise and it has achieved good results.
\end{abstract}

KEYWORDS: Aircraft, Material coding, Material classification.

\section{INTRODUCTION}

In the information age, it is necessary to establish the information system for enterprises to improve their own competitiveness in the increasingly fierce market. Establishing a unified material code of rules is the bottom for enterprises to perform information management. The unreasonable material code would lead to confusion in management. Depending on the rational material coding, we can optimize material management in order to improve the efficiency of management and to reduce the material inventory cost.

It is not, however, an easy work. The study of information classification and coding started in early 1945 , in the United States, and the national material coding system was put forward in 1958. Since the 1960s, countries like Romania and Japan put a large amount of manpower and resources into studying material coding. Recently, many scholars have carried many new researches on how to encode material. Yi et al. (2006) and Wang and Wang (2008) introduced coding technology based on the ontology for information integration. Lei et al. (2008) discussed code principle structures and characteristics of material classification with group technology. Jiang (2007), Wang and Wang (2008) and Zhao et al. (2010) studied the flexible structure and the multi-segment code for the Product Data Management (PDM) system and proposed the information coding model. Material coding management is applied in different industries ( $\mathrm{Li}$ and $\mathrm{Xu}, 2012$; Xiong et al., 2010), and Xiong et al. (2010) suggested that new technologies such as Radio Frequency Identification can be applied in material coding management. Meng and Kong (2013) studied the enterprise material coding in detail, and proposed the purchasing material coding scheme and method,

\footnotetext{
1.Shanghai University - Shanghai - China

Author for correspondence: Hongxia Cai | Shanghai Key Laboratory of Mechanical Automation and Robotics | School of Mechatronic Engineering and Automation | Shangai University | Yanchang Road 149, Zhabei District | 200072 Shanghai/China | Email: hxcai@staff.shu.edu.cn

Received: 12/19/2013 | Accepted: 04/09/2014
} 
based on the K3 auxiliary production software. Wang et al. (2011) put forward the flexible classification and coding system for the rapid design of aircraft tooling components. But there have been few researches on the aircraft material coding, especially for the aircraft manufacturing industry. Due to the characteristics of aircraft material and material management requirements, it is of great importance to study aircraft material coding.

\section{ANALYSIS OF AIRCRAFT MATERIAL CODING}

Material coding, in a few words, is a material management method which uses symbols or numbers in order to represent specifications or categories of materials, so that it is easy to recognize, track and monitor them. These materials cover not only raw materials and parts, but also semi-finished and finished products, purchased parts, packaging materials, product brochures, even including all the tooling resources, labor insurance supplies and energy for manufacturing or services.

As the special discrete manufacturing industry, the materials in the aircraft industry have their own characteristics: various in kinds and quantities, many self-made parts and design changes, strict quality control and tracking measure.
Lots of materials have special customization demand; they must be producted in a single piece or in small batches in order to meet the requirements. Due to its particularity and complexity, material coding is different in the aircraft manufacturing industry.

The general material coding process is shown in Fig. 1. First, sort out all materials. Describe them using a standard pattern and determine the categories for their classification. Then, according to the material classification and its coding standards, it is established a coding structure and a set of encoding rules are generated. Finally, the coding system is developed, so that the material can be automatically coded to be used in the information system.

Because of the particularity of the aircraft manufacturing industry, its material coding presents some difficulties, as shown in Fig. 1, with the red boxes, which are:

\section{AIRCRAFT MATERIAL CLASSIFICATION}

Aircraft material classification should consider the variety of the materials. The material coding process covers almost all departments in the enterprise. The materials have complicated properties, and there is a cross impact between the various materials. How to determine the classification boundary and to make a reasonable classification without any ambiguity to distinguish aircraft material is a difficult task. There are two main classification methods, one is a surface classified method and the other one is line classified. The basic principles of

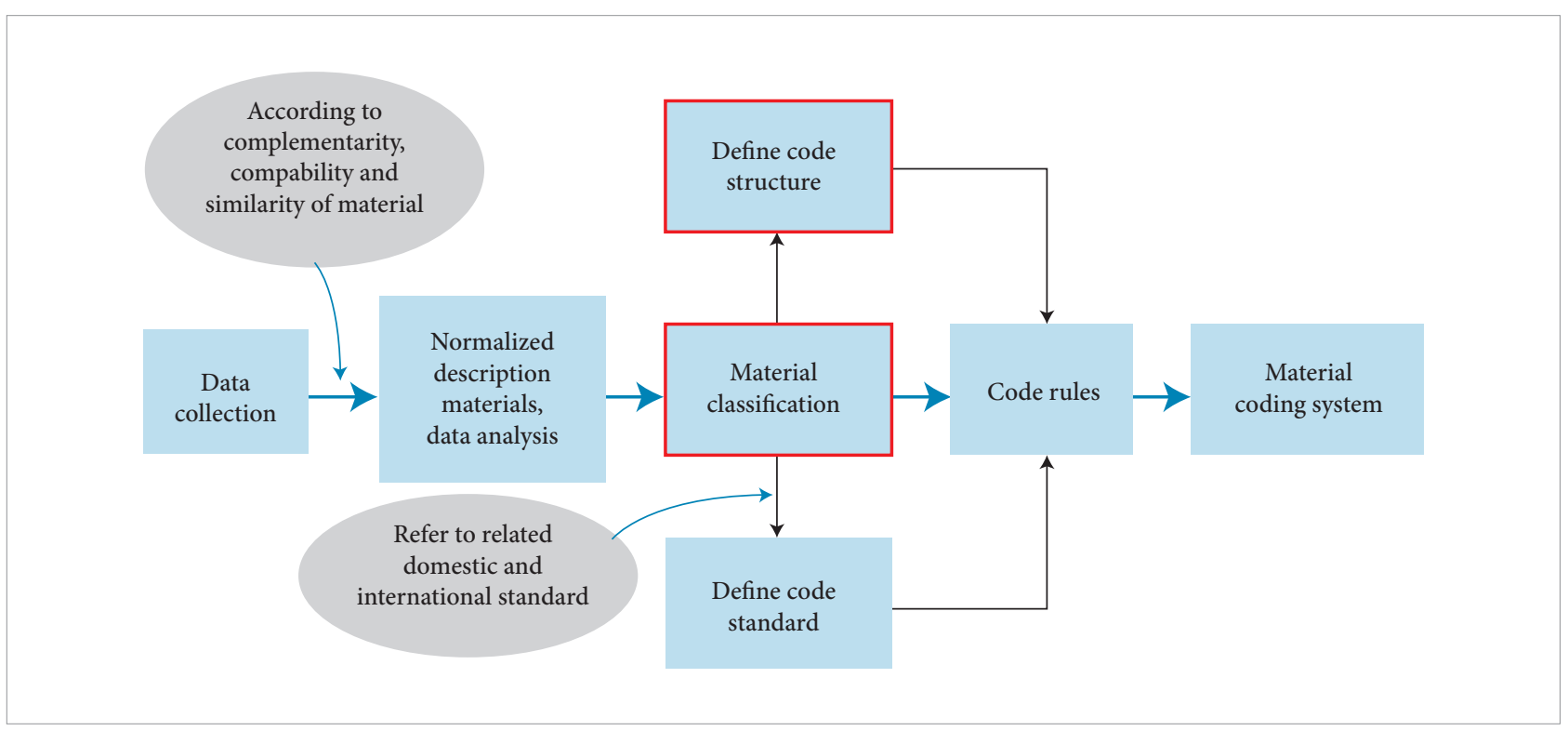

Figure 1. Flow chart of material coding. 
classification are meant to ensure that the classification system embraces the features of comprehension, systematization and expansibility. The material code should be separated into several segments. Taking the overall information of material into consideration and arranging them within the different code segments. Taking the natural material attributes as basis. Reserving enough space to code new attributes, in order to ensure the extensibility of material system.

\section{CODING STRUCTURE AND CODING RULES}

The coding rules should define how long the code is and what information should be included. Because there are so many material properties, it is difficult that all attributes are represented in the material coding. Arguments will be arisen in different departments. The important information of the material is taken into account, while the changeable or configuration information should be avoided. When the length is determined, we should consider reserving the space for the demand of the product expansion, enterprise extension, long-term planning, etc. Of course, the setting of such long material code is discouraged.

\section{MATERIAL CODING SCHEME}

\section{AIRCRAFT MATERIAL CLASSIFICATION}

Material classification is the process of seeking common ground while putting aside differences (Wang and Tong, 2006). It distinguishes and classifies materials through certain principles and techniques, according to properties, attributes or characteristics. In order to make sure high efficiency and convenient maintenance is kept, the material classification should follow the principle of "good compatibility, moderate information capacity, simple and standard".

Taking one aircraft manufacturing company as an example, the statistics show that the aircraft material library is very large. Nowadays, there are more than 100,000 kinds of materials, among which are raw materials, non-metallic materials and composite materials, and so on. The aircraft materials can be classified in different ways based on the requirements. According to the production requirement, they are classified into three categories: large parts (including the fuselage, wing, engine hanger, etc.), aircraft related material, non-production aircraft material (including general consumables, tools, and work clothes, etc.). While in inventory management, the classification is: airborne system finished-products, airborne structural components, metal raw materials, parts, standard parts, auxiliary materials and flying materials (referring to the material used on the plane and being taken away on the plane).

The classification methods above are suitable for materials for aircraft manufacture needs, but they do not include all the materials used in the aircraft manufacturing process. The material code is applied to all production and inventory material in the material coding system. Therefore tools, equipments, fittings and others should be contained. Currently, a simple classification is the $\mathrm{AB}$ classified control method in the aircraft industry. They are divided into two major groups called A and B: group A is the kind of material for aircraft manufacture needs, such as raw material, parts, standard parts, airborne system finished-products, airborne structural components, and so on, and group B consists of auxiliary materials, including tools, equipment, office suppliers, labor protection, etc.

The main research focuses on a detailed classification of group A. Small classes sharing the same characteristics were grouped into one large class based on the analysis from the early chapter, with normal material classification standard. Thus, metal raw materials, chemical materials, parts and standard parts can fall into one class. Group A is divided into five classes: raw material, airborne system finished-products, airborne structural components, self-made parts and equipment delivered together with the aircraft from suppliers. The aircraft classification is shown in Fig. 2 as follows:

\section{AIRCRAFT MATERIAL CODING STRUCTURE}

The material coding structure is generally designed as subordinate relation or material attribute. Also, it can use drawing number as part of the code. The simplest way to do it is sequential coding, which uses Arabic numbers and/ or Latin alphabet letters in sequential order as to identify material, such as the department code. The subornation coding has advantages such as clear structure relations, defined information and being easy to remember, but it does not work well to various types of material. Classification coding is easy to search and control, but hard to determine boundary classification. Sequence code is rarely used alone, because it fails in reflecting the relation between the materials. In recent years, with the increasing study on coding technology, it is 


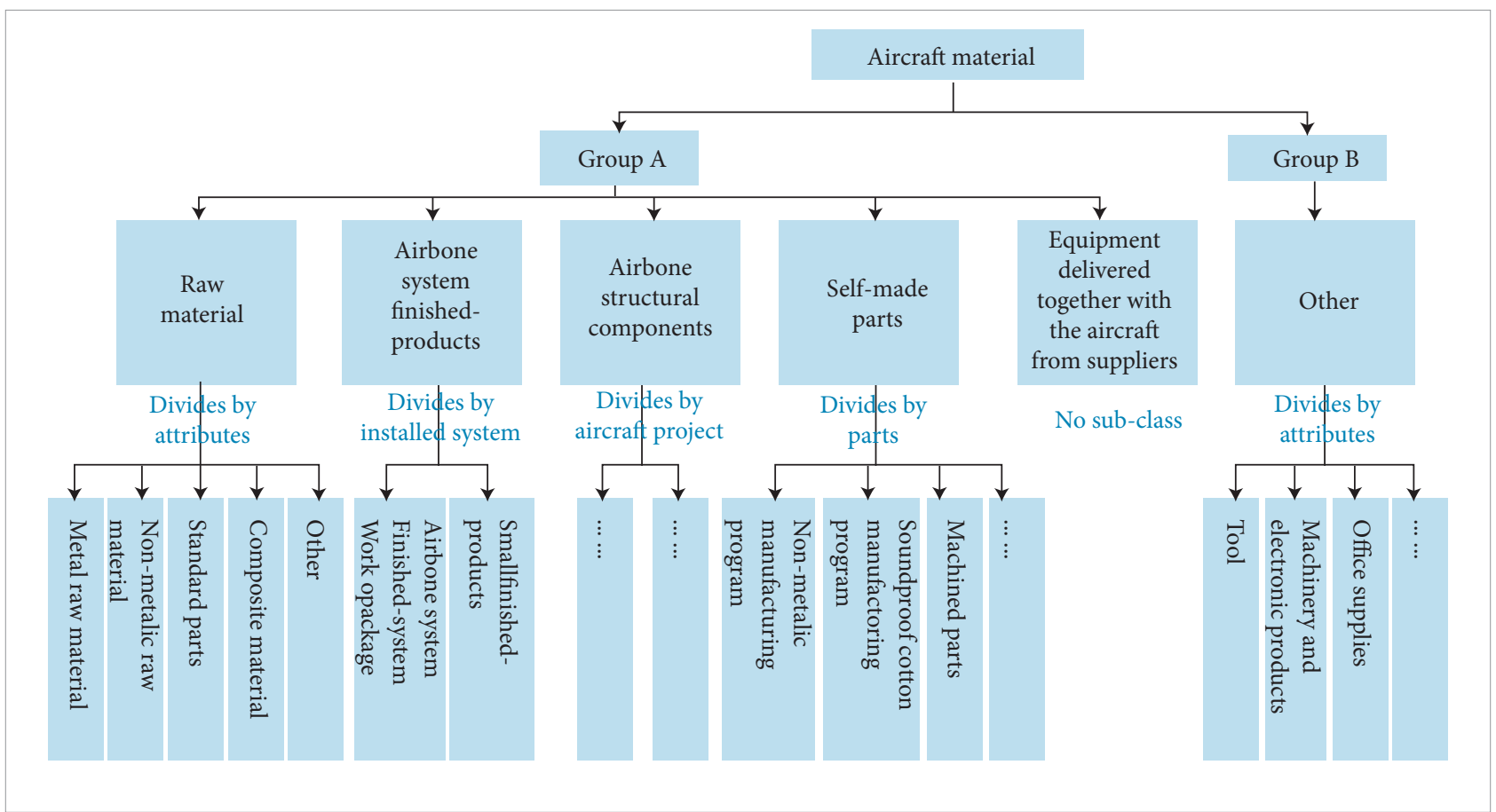

Figure 2. Aircraft material classification.

a trend that code structure is flexible in both code length and code layer relation.

There are some influence factors on flexible code structure. Jiang et al. (2008) evaluated the influence factors of material code structure, including code length, features description, structure stability, standard compatibility, organization rationality and structure uniformity, by using an analytic hierarchy process (AHP). The paper concluded that the material code structure should be designed based on the integration of these six factors. Considering one or a few of them and ignoring the other factors will lead to code structure instability. Therefore, the material code structure should be designed in accordance with the enterprise's material properties and its own requirements. It is not feasible to just copy other enterprises' coding system.

The most common material code structure is defined as: routine code, occupy code or flow code (Yi et al., 2006; Zhao et al., 2010). Based on the aircraft material classification and on essential factors for structure, the composite aircraft code structure is proposed, which consists of three kinds of code fields: class code, property code and flow code, as shown in Fig. 3.

The meta-model is composed of code fields, and the code field relation, in which the code field is the basic unit of code, is classified by the meaning of every code segment, and the code field relation defines the constraint relations among code fields. There are three kinds of code fields: class code, property code and flow code. Based on the classification coding principle, each class code, with the same code structure, has a strong correlation, and it includes class value and class name, which cannot be repeated and contains all material object. Property code is to encode material attributes. Flow code is to distinguish the same kind of material with a sequence of numbers. The initial value and step length of flow code is freely defined. Code fields are not independent and they have constraint among them. There are three relationships in our aircraft material coding structure. The parallel relation is simple, like the same level property code. The subordinate relation refers to generating material code in a certain order. The dependency relationship regulates the generation of code values. Direct dependence is where the front class code decides the later code value. Union dependence is that the flow code relies on the class code and on the property code. The property code depends on the class code, called extensive dependency relation.

The coding structure model is the basis of the code rules. This code structure model can meet the flexible coding requirements with high applicability. 


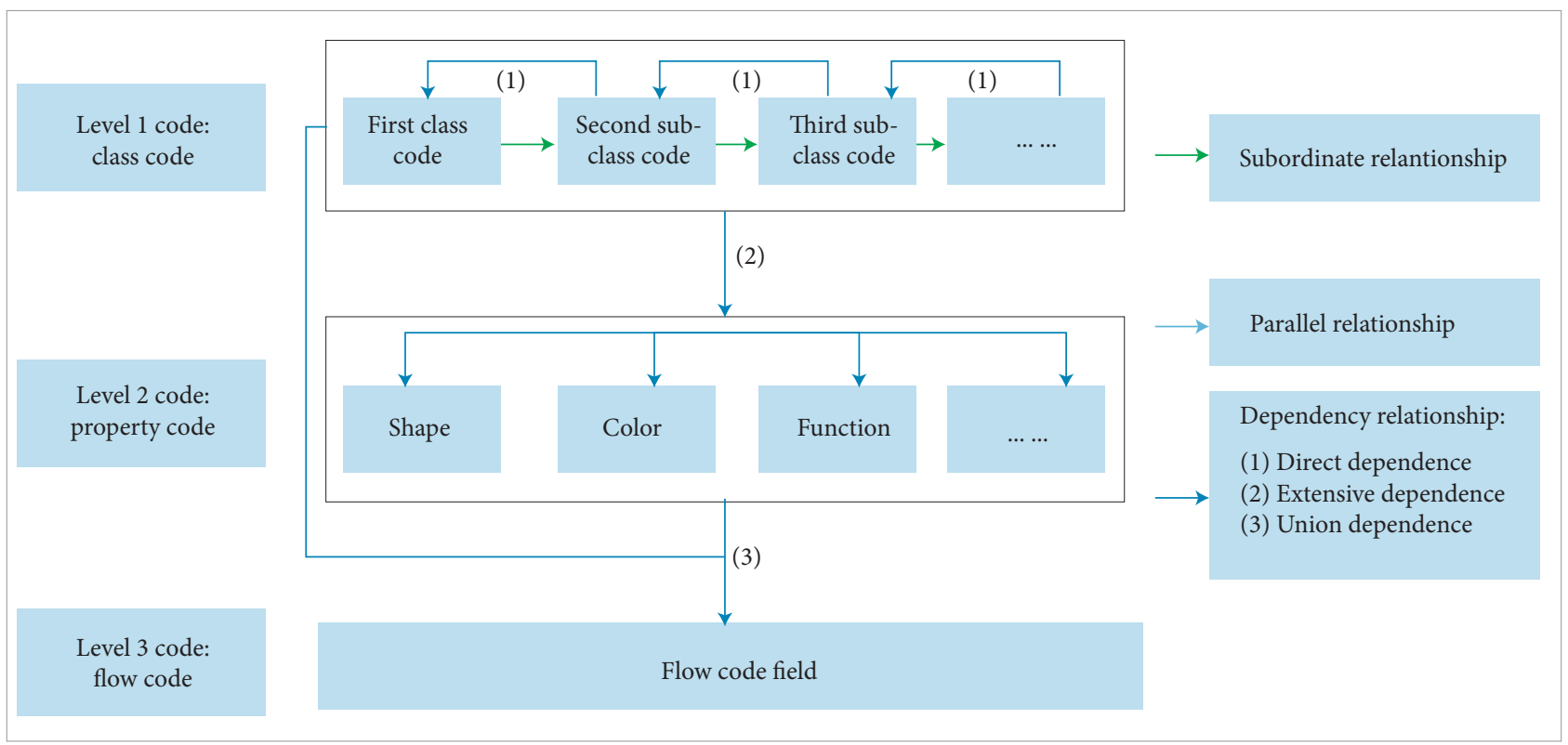

Figure 3. Material code meta-model.

\section{AIRCRAFT MATERIAL CODING RULES}

Material coding should follow some basic principles, such as uniqueness, integrity, stability, simplicity, etc. The aircraft material coding rules is established based on the material coding principles, in reference to the national relevant material coding's standard regulations, while considering both the characteristics of the aircraft industry and the aircraft material features.

Generally, there are three coded forms, the number code, the letter code, and the alpha-numeric code. The number and the letter codes have theirs virtues and shortcomings. The letter code has such advantages as explicit material meaning, convenience of recognition, being easy to remember, but complicated and heavy workload when the material library is large. The number code is easy to operate with, it has quick input capacity, and it obviously improves efficiency, however, it is difficult to identify. The hybrid code method, the alpha-numeric encoding form, takes the best of both approaches and is widely used. The aircraft materials are large and include very complex information. For convenient recognition and operation, the alpha-numeric combination code method is used, with numbers from 0 to 9 and letters from $\mathrm{A}$ to $\mathrm{Z}$, in random permutation.

Based on the code structure and on the alpha-numeric coding form of aircraft material above, the material coding rule is proposed in Fig. 4.
In the aircraft material code rules, the first class code, the second sub-class code, and the last sub-class code length are two bits. One bit cannot satisfy coding requirements, and it represents poor extensibility. While three bits may bring ill consequences, like over long length and a waste of code space. Property code length is different, and we set it according to the different properties of the aircraft material. Flow code length is determined not only to meet the coding requirements, but also to consider the total code length. Code length with a big gap, will bring management trouble. In the first class code, a small number of equipments from the suppliers use the code "U8" to represent them. And other materials in group B are represented with the number " 99 ".

The characteristics above about code rules are that all materials can be well indicated according to category, function, features and attributes. It is scientific and reasonable, avoiding material coding missing and ambiguity.

\section{MATERIAL CODING SYSTEM}

\section{MATERIAL CODING SYSTEM MODEL}

The material coding system mainly consists of three parts: definition of code rules, generation of code value, and code management. 


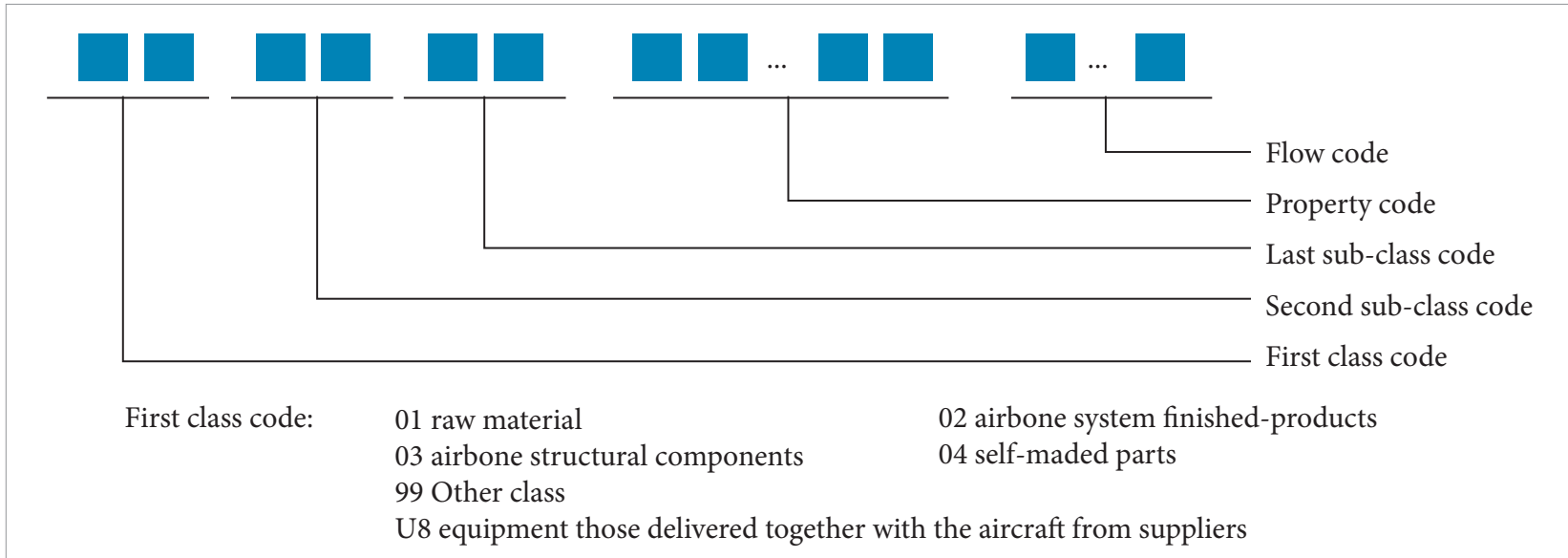

Figure 4. Aircraft material coding rules.

Based on the code structure meta-model, each code field and relation among them should be well-defined in the rule editor. The constraint relationships among code fields should be fixed. Those code rules are put in the code rule library.

Each code should be associated with a corresponding library code. When applying to generate material code, it selects the code rule accordingly, sends the code request to the code generator, and it generates an unique material code. As soon as the code is generated, it is stored in material code library, along with related material information.

Last, the system can enable operators to query or modify code and other management functions in order to maintain conveniece. The aircraft material coding system model is shown in Fig. 5.

\section{KEY CODE TECHNOLOGY}

The key technology of designing coding system is the code metadata model. Based on the code structure meta-model, metadata information model is represented as follows:

The model consists of "Material Object", "Code Rule", "Function" and "Operation". "Material Object" refers to the material and its associated attributes. "Code Rule" includes all defined code rules. "Function" refers to the function of the relation between material and code rule. "Operation" is used to define the code rule's operations. In the model, code rule and code field include their own elements. Code Fields Constraint is the collection of code field relations. Based on the information model, we can build a good database to manage data. Then put it forward in the system processing flow chart in Fig. 6.

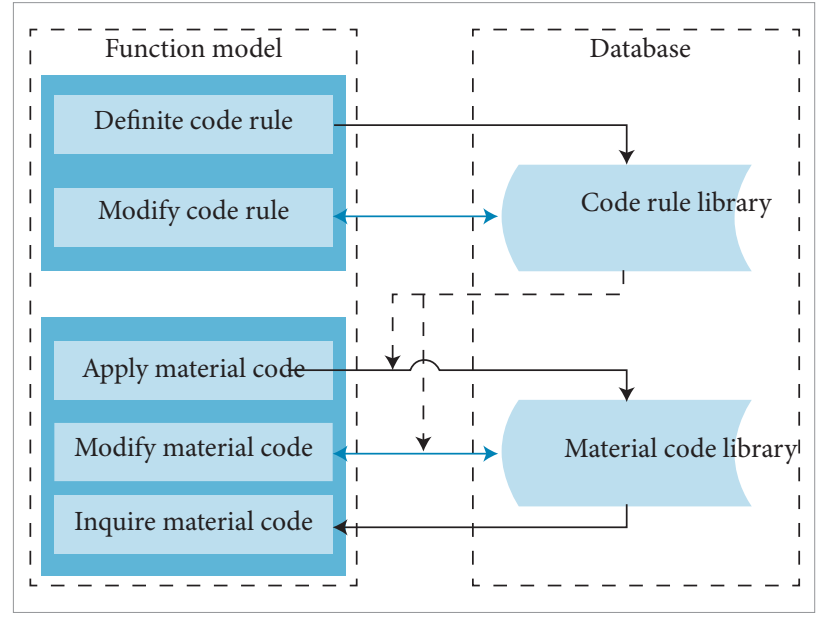

Figure 5. Aircraft material coding system model.

\section{CODING SYSTEM IMPLEMENTATION}

To have a better management of material coding in Enterprise Information systems for the aircraft manufacturing enterprise, the material code management system is developed, with the Java application platform and the Oracle database technology, based on the Browser/Server structure mode. The system could generate the material code automatically, inquire the material code, search material, and manage material classification. Some main functions and interfaces of the system are discussed below.

\section{Material code application}

Input material information and select material classification from the drop-down list shown in the page (Fig. 7). Use Ajax techniques to refresh only material classification items instead of 


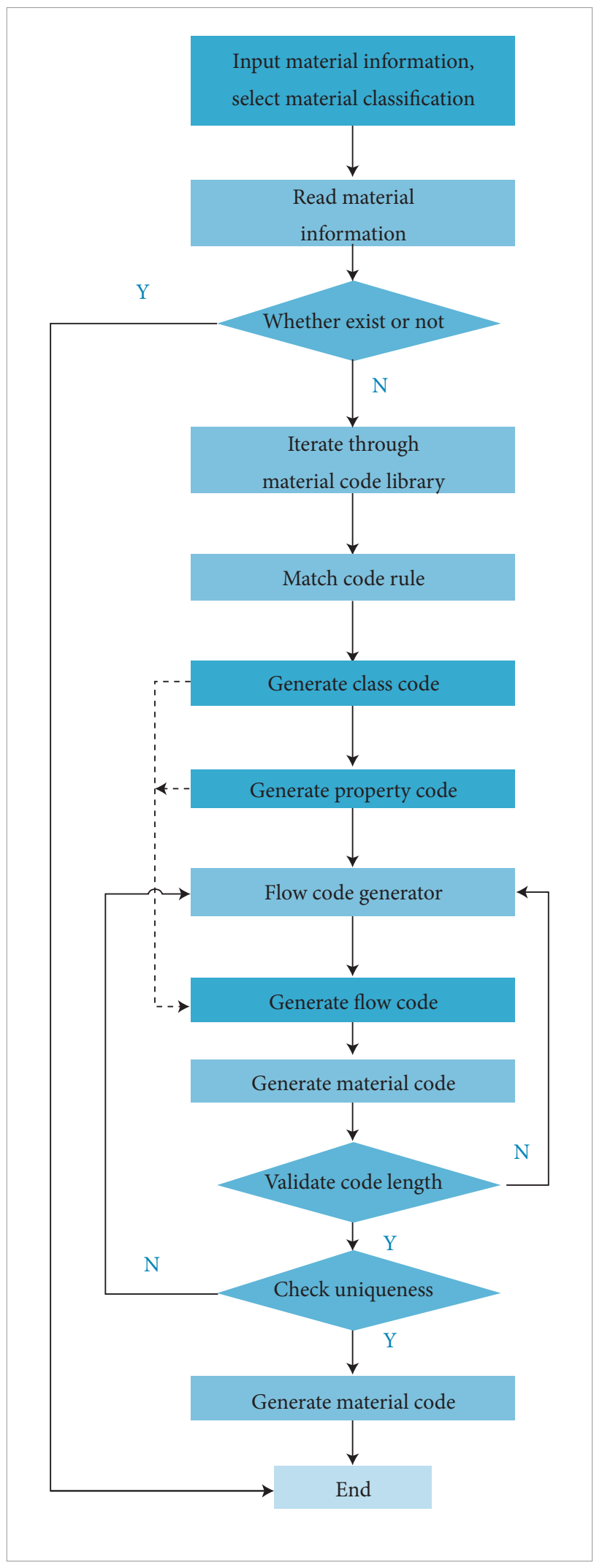

Figure 6. System processing flow chart. the whole page. When the first class is selected, decide whether the sub-class exits or not, and then update dynamically and display sub-class items.

\section{Material code query}

Select the search criteria, such as "classification", "material code", "applicant", show the result in list (Fig. 8). The status is important, which shows the material code is either approved or waiting for approval. And the approver can edit it by clicking the button "approve" or "reject". Through the column "application path", the material classification is easy to be identified.

\section{Material query}

This search interface (Fig. 9) shows material information, including material code, name and so on. By selecting the search criteria, those materials which need to be managed and edited are found.

\section{CONCLUSIONS}

Material coding is of great significance for the enterprise information management. Taking the characteristic of aircraft materials into consideration, the paper studies material coding technology and proposes an aircraft material coding scheme. Firstly, it classifies aircraft material, secondly establishes code structure and code rule, and finally develops the material coding system which has been applied in the aircraft manufacturing enterprise and achieved good results. This study has greatly improved the coding efficiency and provided a classification method for aircraft material coding and powerful support in this aspect.

\section{ACKNOWLEDGMENT}

This work is supported by the Shanghai Science and Technology Committee under Grant No. 12dz1124300\& 13521103604. The authors are grateful for the financial support, and they also would like to thank the anonymous reviewers and the editor for theirs comments and suggestions. 
Cai, H. X., Dai, M. Y. and Yu, T.

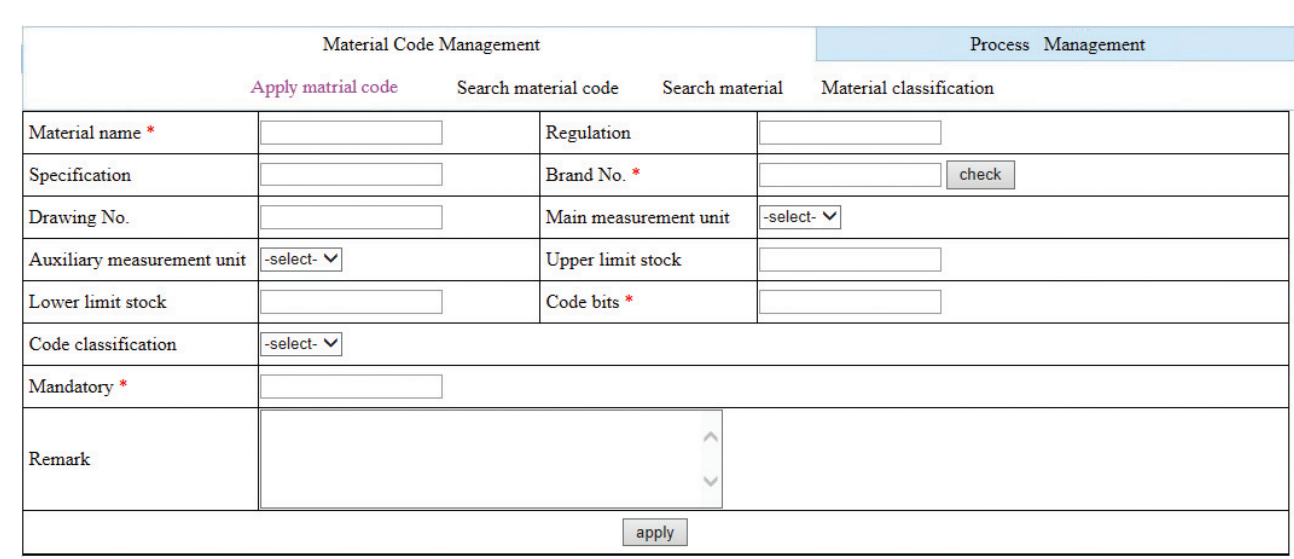

Figure 7. The material code application interface.

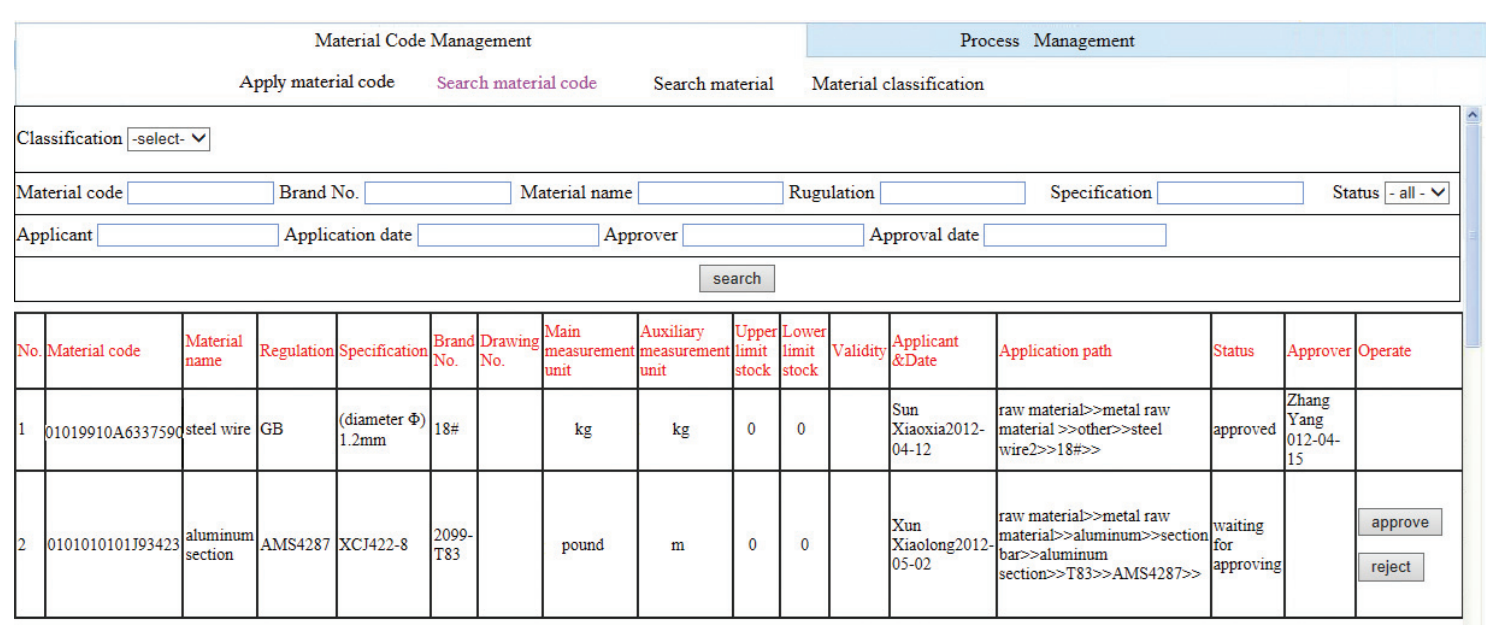

Figure 8. The material code query interface.

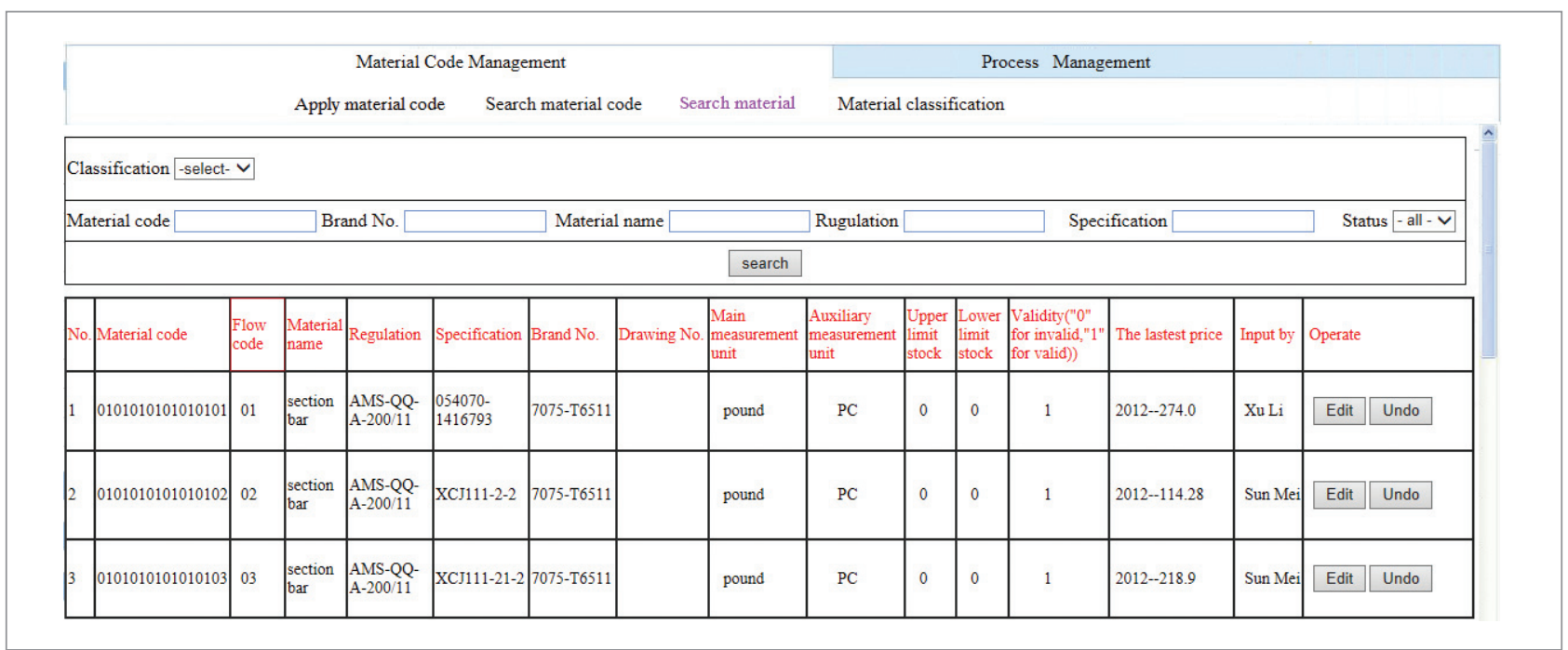

Figure 9. The material query interface. 


\section{REFERENCES}

Jiang, J., 2007, "An Effective Method Based on Granularity-Structure for Organizing and Utilizing Manufacturing Information Resource", Journal of Northwestern Polytechnical University, Vol.25, No.2, pp. 245-250.

Jiang, J., Wang, J., Guo, L. and Liu, Y., 2008, "Research on Unified Coding Structural Model of Manufacture", Mechanical Science and Technology for Aerospace Engineering, Vol.27, No.1, pp. 13-18 (in Chinese).

Lei, W., Yang, C. and Li, Y., 2008, "The Material Classification Codes System", Group Technology \& Production Modernization, Vol.25, No.1, pp. 60-63 (in Chinese).

Li, Y. and Xu, D., 2012, "Development of cutting tools information coding rules for the tools management system", Proceedings - ICIDT 2012, 8th International Conference on Information Science and Digital Content Technology, Vol.2, pp. 397-400.

Meng, X. and Kong, Y., 2013, "Material Classification and Coding based on K3 Manufacture Assistant Software", Shanghai Chemical Industry, Vol.38, No.2, pp.19-21 (in Chinese).

Wang, J. and Wang, B., 2008, "Research on Application of Ontological Information Coding in Information Integration", Global Design to Gain a Competitive Edge, pp.147-155. doi: 10.1007/978-1-84800-239-5_15.
Wang, K. and Tong, S., 2006, "Manufacturing Quality Information Classification based on Group Technology and Quality BOM", IMACS, Multi-conference on "Computational Engineering in Systems Application", pp. 2160-2167.

Wang, R., Zhou, L. and An, L., 2011, "Research on Flexible Classification and Coding Technology for Airplane Fixture Components", Mechanical Engineering \& Automation, Vol.1, pp. 2123 (in Chinese).

Xiong, G., Hu, L., Qin, T., Nyberg, T.R., Wang, F. and Shi Q., 2010, "Design and Improvement of the Material Coding Standardization for Power Group Enterprise", IEEE International Conference on Automation and Logistics, ICAL, pp. 597-602.doi: 10.1109/ ICAL.2010.5585353.

Yi, J., Pan, P. and Dong, J., 2006, "Ontology- based PDM coding management middleware", Computer Integrated Manufacturing Systems, Vol.12, No.6, pp.1821-1826 (in Chinese).

Zhao, H., Liu, J., Dong, Y. and Xu, Z., 2010, "Research on Flexible Coding System Model for PDM System", Journal of Engineering Graphics, Vol. 31, No. 5, pp. 34-38 (in Chinese). 